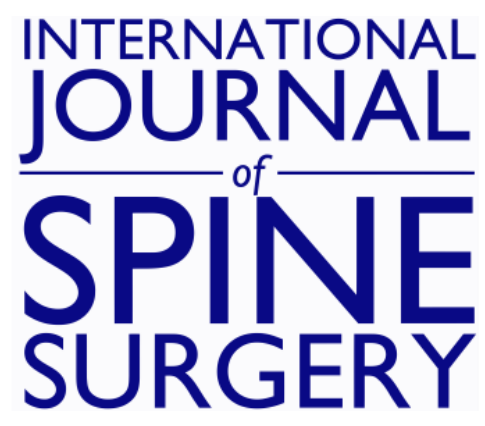

\title{
Nubac Disc Arthroplasty: Preclinical Studies and Preliminary Safety and Efficacy Evaluations
}

Qi-Bin Bao, Matthew Songer, Luis Pimenta, Dieter Werner, Alejandro Reyes-Sanchez, Massimo Balsano, Umberto Agrillo, Domagoj Coric, Kenneth Davenport and Hansen Yuan

Int J Spine Surg 2007, 1 (1) 36-45

doi: https://doi.org/10.1016/SASJ-2006-0007-RR

http://ijssurgery.com/content/1/1/36

This information is current as of April 26, 2023.

Email Alerts Receive free email-alerts when new articles cite this article. Sign up at:

http://ijssurgery.com/alerts

The International Journal of Spine Surgery

2397 Waterbury Circle, Suite 1,

Aurora, IL 60504, Phone: +1-630-375-1432

(C) 2007 ISASS. All Righfso Refservededom http://ijssurgery.com/ by guest on April 26, 2023 


\title{
Nubac Disc Arthroplasty: Preclinical Studies and Preliminary Safety and Efficacy Evaluations
}

Qi-Bin Bao, PhD, Matthew Songer, MD, Luis Pimenta, MD, PhD, Dieter Werner, MD, Alejandro Reyes-Sanchez, MD,

Massimo Balsano, MD, Umberto Agrillo, MD, Domagoj Coric, MD, Kenneth Davenport, MD, and Hansen Yuan, MD

\begin{abstract}
Background

Disc arthroplasty is gaining popularity for treatment of low-back pain caused by degenerative disc disease (DDD). It can involve total disc replacement or partial disc or nucleus replacement (or augmentation). Compared with total disc replacement, nucleus replacement is less invasive, has less surgical risk, has faster postoperative recovery, and doesn't "burn bridges" should further surgery be required. However, nucleus replacement has a high risk of implant expulsion because the device is not fixed to the vertebrae. Nubac is the first polyetheretherketone (PEEK)-on-PEEK articulated disc arthroplasty device designed to optimally restore the lumbar anatomy and biomechanics.
\end{abstract}

\section{Methods}

ISO 10993 standards were used to evaluate the biocompatibility of the PEEK material. Chemical and thermal-mechanical tests and in vivo study assessed PEEK's biostability after exposure to high g irradiation and harsh oxidative conditions. Biomechanical tests to evaluate kinematic properties and anatomical restoration of the implanted lumbar motion segments and implant expulsion risk assessments were performed with a human cadaveric model. Because of the novelty of PEEK-on-PEEK as a self-mating articulating material, extensive wear tests were conducted with unidirectional and coupled motions. Static and fatigue strength also were tested. Animal study with a baboon model was conducted with gross, radiographic, biomechanical, and histological evaluations at 6 and 12 months postoperatively. Preliminary clinical data were collected through a prospective multicenter cohort study.

\section{Results}

PEEK demonstrated exceptional biocompatibility and biodurability. Nubac restored disc height and motion segment range of motion. The unique articulating design of the Nubac demonstrated low risk of implant expulsion in a human cadaveric model. Wear tests showed that the Nubac has minimal wear and compares favorably to other disc arthroplasty materials. The Nubac also had excellent static and fatigue properties for the intended application. The animal study showed that the Nubac caused no adverse local or systematic tissue reaction and there was no detectable wear debris. The preliminary clinical data showed no major intraoperative vascular and neurological complications. There was significant Visual Analog Scale and Oswestry Disability Index score improvement.

\section{Conclusions}

The preclinical data supported the design rationale, and the preliminary clinical data (level II evidence) on safety and efficacy were encouraging.

\section{Clinical Relevance}

The Nubac could be a viable first surgical option for patients with back pain caused by DDD.

Key Words disc arthroplasty, nucleus replacement, PEEK-on-PEEK articulation. SAS Journal. Winter 2007; 1; 36-45. DOI: SASJ-2006-0007-RR

\section{INTRODUCTION}

Disc arthroplasty is gaining popularity for the treatment of lowback pain caused by degenerative disc disease (DDD). Disc arthroplasty includes both total disc replacement and partial disc or nucleus replacement (or augmentation). Compared with total disc replacement, nucleus replacement has the advantages of being less invasive, having less surgical risk, and having faster postoperative recovery; it doesn't "burn bridges" should further surgical treatment be required. However, the main challenge of 
nucleus replacement has been its high risk of implant expulsion because the device is not fixed to the vertebrae.

The purposes of this study were to verify the design rationale of Nubac (Pioneer Surgical Technology, Marquette, Mich) via a series of preclinical studies either internally or in collaboration with outside institutions and to give a preliminary assessment on the safety and effectiveness of Nubac from a prospective cohort study. The design rationale of the Nubac intradiscal arthroplasty device was largely based on the intended clinical objectives of an intradiscal arthroplasty device and the clinical history of disc arthroplasty devices. Clinically, an intradiscal arthroplasty device is mainly designed to treat patients with discogenic back pain caused by DDD. Because discogenic back pain can be caused by a chemical origin and/or mechanical irritation/ instability, in the absence of a definite diagnostic method to determine the exact pain mechanism, an intradiscal arthroplasty procedure must address both potential pain mechanisms.

It is hypothesized that by removing the "diseased" nucleus and replacing it with an intradiscal arthroplasty device, chemical pain can be relieved. It is well known that removal of the nucleus will cause collapse of the disc height and lead to further instability of the index segment, which in turn can cause mechanical pain. It is further hypothesized that an intradiscal arthroplasty device can maintain or restore the disc height and mechanical function of the natural nucleus and therefore relieve and/or prevent mechanical pain. Mechanically, in a healthy disc, the nucleus shares the compressive load with the annulus, taking about half of the total load passing through the anterior column. ${ }^{1}$ Because of its hydrostatic nature, the nucleus distributes the load evenly over the endplates under all physiological loading conditions ${ }^{2}$ and presents no restriction to the rotational motion by itself. The resistance to rotation relies mainly on the annulus and the facet joints.

For total disc arthroplasty, the ball-and-socket articulation has become the most popular design and has shown its ability to restore the physiological range of motion (ROM) in flexion/ extension and lateral bending directions. Clinically, total disc replacements that incorporate a ball-and-socket design have shown reasonably good results, both in relieving pain and maintaining ROM. ${ }^{2-4}$ The designs and materials for nucleus arthroplasty, however, vary from hydrogel or nonhydrogel elastomers, which are either preformed or formed in situ, to nonelastomeric materials such as metal, polymethyl methacrylate (PMMA), and pyrolytic carbon. None of these nucleus devices has yet to incorporate an inner articulation design.

Clinically, the early Fernstrom ball, which had bipolar articulation with the endplates, showed fairly good short-term and long-term results. ${ }^{5,6}$ The main criticism of the Fernstrom ball was its high rate of implant subsidence. This was because the implant has an initial point contact with the endplates, leading to high contact stress and, therefore, subsidence. As the implant subsides into the vertebrae, the contact area increases and the contact stress decreases. Eventually, the contact stress will reach a point that is below the critical stress and subsequently the subsidence risk becomes relatively low. It has been reported that most of the Fernstrom devices had $1 \mathrm{~mm}$ to $3 \mathrm{~mm}$ of subsidence on each side. ${ }^{6}$ Taking a $12-\mathrm{mm}$ diameter Fernstrom ball (the diameter range of Fernstrom is $10 \mathrm{~mm}$ to 16 $\mathrm{mm}$ ) as an example, the projected contact cross-section area is $85 \mathrm{~mm} 2$ after $3 \mathrm{~mm}$ subsidence. Therefore, if a device with an initial contact area is larger than this value, the subsidence risk should be relatively low if the implant height is properly chosen to achieve adequate load sharing between the nucleus device and the annulus.

It should be pointed out that regardless of the amount of subsidence, the clinical outcome for the Fernstrom ball, in both the short term and long term, was fairly good, with more than $75 \%$ of patients reported to be "good" or "excellent." ${ }_{5,6}$ It was also reported that ROM was observed after the implantation of the Fernstrom ball. This favorable clinical outcome, compared to that of PMMA as a nucleus replacement, should be largely attributable to its biconvex feature that allows more uniform stress distribution under various physiological rotational motions.

Being based on the design objectives and the lessons learned from various previous disc arthroplasty devices, the design of the Nubac includes all the major benefits of both total disc arthroplasty and nucleus arthroplasty, while eliminating the major pitfalls of these designs. It has a unique 2-piece design with an inner ball-and-socket articulation. The implant is made of polyetheretherketone (PEEK)-Optima (Invibio Ltd, West Conshohocken, Pa), which has well-established biocompatibility, superb biodurability, and a history of use as a permanent spinal implant.

The outer surface of each piece has an oval (ice rink) shape to mimic the general shape of the nucleus and a large contact surface for the endplates. This large contact area is designed to distribute load, reduce contact stress, and thereby mitigate the risk of subsidence. For example, the smallest footprint available has a contact surface area 2.2 times that of the contact area of a 12-mm Fernstrom ball device after $3 \mathrm{~mm}$ of subsidence. Therefore, the risk of subsidence of the Nubac should be relatively low.

The Nubac intradiscal arthroplasty device retains the advantages of nucleus devices, being both less invasive and a less of a bridge-burning procedure than total disc arthroplasty devices. However, like many total disc arthroplasty devices, 
it has an inner articulation that brings several advantages over nonarticulating nucleus arthroplasty devices. Therefore, the Nubac to some degree is a hybrid device and forms a unique category as an intradiscal arthroplasty device, for the following reasons. First, like the natural nucleus tissue, it does not restrict any physiological rotational motions. It should be emphasized that, unlike total disc replacement, the constraint (i.e., stability) of the motion segment after an intradiscal or nucleus arthroplasty procedure is largely determined by the surrounding tissues, such as the annulus and ligaments. Because most of these surrounding tissues are preserved in the intradiscal arthroplasty procedure, and the annulus is restored to the normal tension stage, the segment mobility and stability after the Nubac implantation is maintained. Second, the device is designed to allow more uniform stress distribution on the endplates under all physiological rotational motions (Figure 1). Third, this design might also reduce the risk of implant expulsion, because as the segment bends opposite to the annular window (this is required for implant insertion) the dimension of the Nubac near the annular window increases and makes expulsion more difficult (Figure 1).

Figure 1

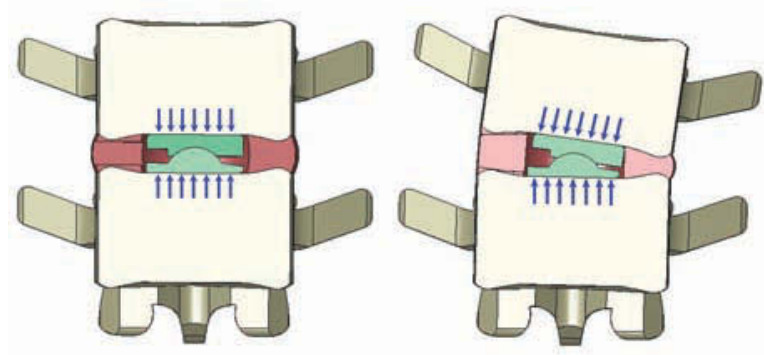

Schematic drawings of stress distribution under compression and bending: (a) Nubac nucleus device under compression; (b) Nubac nucleus device under bending.

In selecting the appropriate material for the Nubac, a novel articulating/material combination of PEEK on PEEK was chosen. Historically, metal $(\mathrm{CoCr})$ on ultra-high-molecularweight polyethylene has been the predominant articulating material combination for orthopedic load-bearing arthroplasty devices, followed by metal on metal and a few other lessfrequently used material combinations, such as ceramic on ceramic. The wear and durability of ultra-high-molecularweight polyethylene for the total joint applications, which are typically used for patients older than 65 years, has been well studied, but the limiting factor, osteolysis, has led to early implant failure. Although osteolysis has yet to become a major clinical problem in total disc arthroplasty, the long-term wear and durability of ultra-high-molecular-weight polyethylene has not been well established for disc arthroplasty applications. This is especially important because the patient population for disc arthroplasty is much younger than that for total joint arthroplasty.

Although metal on metal has better durability, the long-term effects of metal ion release have not been fully studied. Ideally, intradiscal arthroplasty devices should be manufactured from nonmetallic material(s) to minimize interference with conventional radiographic imaging techniques, such as magnetic resonance imaging and computed tomography. PEEK is one of the best thermoplastic materials and has well-established biocompatibility and superb mechanical and biostability characteristics. Because many patients with DDD are aged 30-50 years, it is especially important to use a material that has exceptional durability.

\section{MATERIALS AND METHODS Biocompatibility and Biodurability}

Independent laboratories have performed biocompatibility and biostability testing on PEEK-Optima relevant to the International Organization for Standardization's ISO 109937 procedures. Invibio, the manufacturer of PEEK-Optima, has conducted extensive biodurability studies. In one study, PEEKOptima was subjected to $200 \mathrm{kGy} \mathrm{g}$ irradiation followed with accelerated aging in oxygen $\left(40\right.$ days at 5 bar at $\left.70^{\circ} \mathrm{C}\right) .{ }^{8}$ In another in vivo biodurability study, samples of PEEKOptima extruded rod were g sterilized with a dose of 73.2 $\mathrm{kGy}$ and then incubated in physiological saline for 3 months at $90^{\circ} \mathrm{C}$ (simulation of 10 years real-time aging at $37^{\circ} \mathrm{C}$ ). ${ }^{8}$ The PEEK-Optima samples were then implanted in an animal model for a period of 12 months and subjected to cytotoxicity testing (ISO 10993-5), chemical analysis (ISO 10993-18), and histopathological examination. ${ }^{8}$

\section{Kinematic Tests}

Two separate kinematic tests, each with somewhat different emphases, were conducted with a human cadaveric model. The first study emphasized the effect of the Nubac on the multidirectional ROM and neutral zone. An in vitro biomechanical study was undertaken to compare the multidirectional flexibility kinematics of the articulating Nubac intradiscal arthroplasty device for single-level reconstruction. A total of 8 human cadaveric lumbar spines (L2-L3 and L4-L5 segments) were nondestructively evaluated under the following conditions: (1) intact spine, (2) nucleotomy alone, and (3) Nubac implant. Unconstrained intact moments of $\pm 7.5 \mathrm{Nm}$ were used for axial rotation, flexion/extension, and lateral bending testing, with quantification of the operative level ROM (degrees) and neutral zone (degrees). A $10 \mathrm{~mm} \_10 \mathrm{~mm}$ annular window was created in each specimen with a right lateral approach, followed by a complete nucleotomy and insertion of the Nubac device.

The second study emphasized the effect of the Nubac on the disc height change and fatigue/expulsion behavior. It examined 
the following performance characteristics of the Nubac device: disc height change, ROM, and stiffness (motion resistance). Six adjacent pairs of human cadaver lumbar functional spine units (L3-L5) were tested at 3 different stages (intact, postdiscectomy, and postnucleus implant) under various physiological loading conditions: $1200 \mathrm{~N}$ was used for compression, and a 7.5 $\mathrm{Nm}$ moment was used for all rotational tests with a $500 \mathrm{~N}$ compressive load. One disc level of each specimen was left intact and served as a control level and the second level served as the surgical level. A $6 \mathrm{~mm}$ _ $10 \mathrm{~mm}$ annular window was created in each specimen with a right lateral approach followed by a complete nucleotomy and insertion of the Nubac device.

\section{Expulsion Tests}

This second kinematic study went on to test expulsion risk. After the nondestructive testing, the same 6 adjacent pairs of human cadaver lumbar functional spine units (L3-L5) were tested with 100,000 cycles of unilateral left bending opposite to the annular window under $2.5-7.5 \mathrm{Nm}$ at $2 \mathrm{~Hz}$ with the compressive load ranging between $250 \mathrm{~N}$ and $750 \mathrm{~N}$. Under this loading condition, the average bending angle was $4.4^{\circ}$ at the beginning of the test and $9.2^{\circ}$ at 100,000 cycles.

Another cadaver study was conducted to assess implant expulsion risk as part of assessing the risks and benefits of different outer surfaces for the Nubac implants. This began with hand-bending the implanted functional spine units after a normal surgical procedure and progressed to include entirely removing the annulus and testing in a materials testing machine (MTS Systems Corp, Eden Prairie, Minn) with lateral bending at approximately $7^{\circ}$ of bending angle. A total of 5 human cadaver lumbar functional spine units were used.

\section{Wear Tests}

In the first test (conducted by Pioneer), 6 of the smallest size of the Nubac implant (which have the smallest contact area and, therefore, the highest contact stress) were used. The samples were subjected to a dynamic axial load of between $225 \mathrm{~N}$ and $1024 \mathrm{~N}$ at $4 \mathrm{~Hz}$ coupled with a flexion/extension rotation with a total of $15^{\circ}$ at $2 \mathrm{~Hz}$ in such a way that peak load coincided with the maximum rotation angle. The load magnitude was chosen on the basis of the design goal of having the intradiscal arthroplasty device share the compressive load with the annulus. ${ }^{1}$ The ROM in this test represents the high end of the physiological flexion/extension for the lumbar discs. The test samples were bathed in $37^{\circ} \mathrm{C} \pm 3^{\circ} \mathrm{C}$ newborn calf serum and the test was conducted to 10 million cycles. At approximately every 500,000 cycles, the test was stopped and all components were cleaned and the specimens weighed to assess mass loss and wear rate. The test fluid was also changed and stored at $-20^{\circ} \mathrm{C}$ for particle analysis. To allow for a more comprehensive assessment of wear and mechanical durability under coupled motion, the devices were rotated $90^{\circ}$ to simulate a lateral bending profile and tested under the same load magnitude and ROM for an additional 10 million cycles. To address the longterm wear durability for the disc arthroplasty devices, the wear test for the Nubac has been further extended to a total of 40 million cycles with alternating flexion/extension and lateral bending.

The size and morphology of the wear debris for every sample were analyzed with enzymatic digestion of the testing fluid, and then subjected to laser diffraction at 0.5 million cycles and at every million cycles thereafter (1.0, 2.0, etc). Scanning electron microscopy was also performed on some representative samples at 0.5 million cycles and at every odd million-cycle count thereafter $(1.0,3.0$, etc).

To assess the coupled motion effect on the wear characteristics of the Nubac device, a second wear test, using the same conditions as the ISO standard9 for total disc arthroplasty, was conducted with an Endolab spine implant testing machine (EndoLab Mechanical Engineering GmbH, Rosenheim, Germany) except that the dynamic compressive magnitude was adjusted to $225 \mathrm{~N}$ to $1024 \mathrm{~N}$ to reflect the load-sharing mechanism for a nucleus device. Again, 6 of the smallest size of the Nubac implant were used.

\section{Static and Fatigue Strength Test}

Six of the smallest size of the Nubac implant, which represents the worst-case scenario in terms of stresses, were bench-tested in an axial static compressive mode until failure to establish the device's static strength. A total of 6 implants were tested by Pioneer under 3 different loads in axial dynamic fatigue mode at $10 \mathrm{~Hz}$ to establish the device's fatigue strength.

\section{Animal Study}

An animal study with a baboon model was conducted to assess the safety of the Nubac device and to perform a limited assessment of its efficacy. Although using a baboon model to assess human disc arthroplasty devices involves limitations (described in Results), the baboon is considered one of the best models available.

The study population was 14 mature male baboons (Papio cynocephalus) randomized into 2 postoperative time periods of 6 months $(n=7)$ and 12 months $(n=7)$. Comprehensive analyses including gross necropsy, magnetic resonance imaging, plain $\mathrm{X}$ ray and microradiographies, multidirection biomechanical tests, and biocompatibility assays with local and systemic histology and immunohistochemistry, were performed at 6 and 12 months after the operation. Each animal underwent a lateral transperitoneal surgical approach followed by complete nucleotomy at L3-L4 and L5-L6 levels. The L5-L6 level was reconstructed with the Nubac device. The L3-L4 served as a surgical (nucleotomy) control in each case. 


\section{Clinical Study}

After extensive preclinical testing, a prospective multicenter clinical study was initiated (level II evidence). The main indication for Nubac is discogenic back pain, with or without leg pain, caused by DDD as evidenced by a dark disc found in magnetic resonance imaging and/or disc height loss. Essentially, all study sites followed a similar study protocol as to the patient indications. Depending on surgeon preferences and patient pathologies, 3 different surgical approaches were used: posterior, lateral, and anterolateral. Most of the patients in this cohort study underwent single-level procedures, with a few 2-level procedures. Oswestry Disability Index10 (ODI) and Visual Analog Scale (VAS) scores were collected preoperatively and 6 weeks, 3 months, 6 months, 12 months, and 24 months postoperatively.

\section{RESULTS}

\section{Biocompatibility and Biodurability}

Biocompatibility testing showed excellent results and the PEEK-Optima m material passed all ISO-10993 tests. Device and drug master files on file with the US Food and Drug Administration contain these results, as well as results from additional testing and extensive data concerning the polymer and its manufacturing methods. It was found that even after a high dosage of $\mathrm{g}$ irradiation and extensive aging, there was no significant change in material mechanical properties, as verified by Fourier transform infrared spectroscopy, gel permeation chromatography, and differential scanning calorimetry. ${ }^{8}$ The in vivo biodurability study confirmed the material to be noncytotoxic, and no obvious degradation, with no muscle necroses, marked inflammatory responses, or any other significant changes, was found. ${ }^{8}$

\section{Kinematic Tests}

In the first study, multidirectional flexibility testing indicated significant increases in the segmental ROM and neutral zone secondary to the annulotomy/nucleotomy procedures, according to repeated measurements (analyses of variance). For both calculated parameters, segmental rotation increased for the destabilized condition versus the intact and reconstructed specimens $(P<.05)$. For the Nubac-reconstructed condition, the neutral zone - an indicator of spinal stability - returned to levels not statistically different from the intact condition. Overall, the use of the articulating Nubac device reestablished the operativelevel kinematics to the intact condition (Figures 2 and 3).

The second study showed that disc height after discectomy (without implant) significantly decreased $(P<.05)$ compared with the intact condition under $1.2 \mathrm{kN}$ of compressive loading. Implantation of the Nubac intradiscal arthroplasty device was able to restore the disc height to the intact stage with no significant difference between the intact and implanted
Figure 2

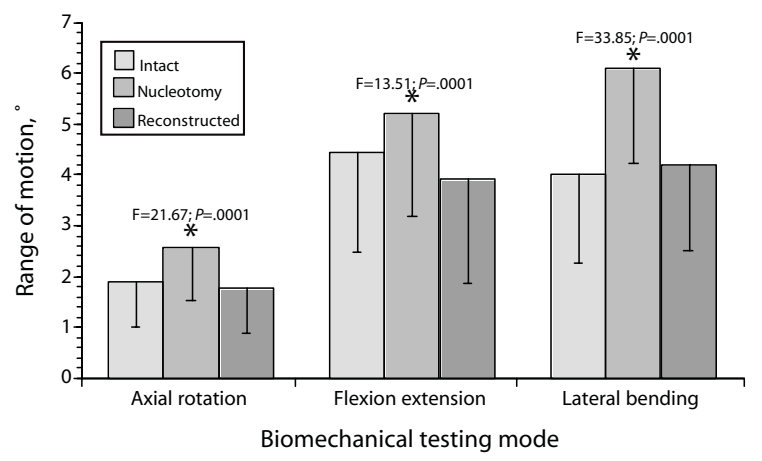

*Indicates statistical difference between the nucleotomy condition versus the intact and reconstructed conditions at $\mathrm{P}<.05$. No other differences were observed. Error bars indicate 1 standard deviation.

Operative-level range of motion.

Figure 3

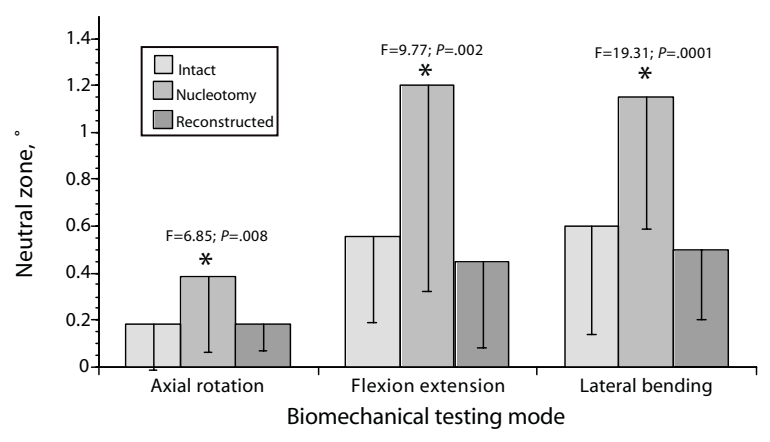

*Indicates statistical difference between the nucleotomy condition versus the intact and reconstructed conditions at $\mathrm{P}<.05$. No other differences were observed. Error bars indicate 1 standard deviation.

Operative-level neutral zone.

conditions (Figure 4). Maintaining or restoring the disc height at the index level is important for balancing the load distribution between the anterior column and posterior column and maintaining the opening of foramen space.

For ROM testing, control levels displayed consistent motion for all modes throughout testing. There were no significant differences in torsional motion for any disc condition. The surgical levels showed increased flexion motion following discectomy (without implant), but this increase was not statistically significant. For extension, the motion 
Figure 4

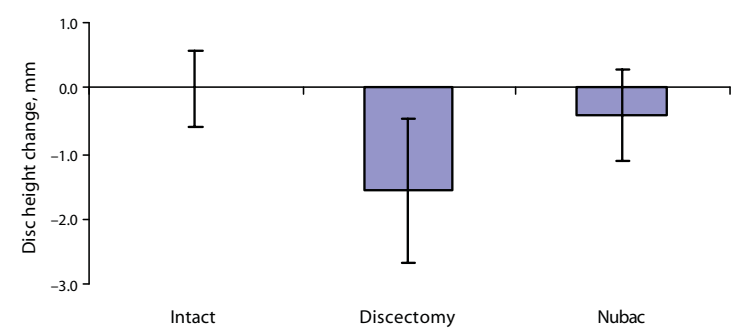

Disc height change after nucleotomy and Nubac implantation.

was significantly reduced $(P<.05)$ after discectomy, but was restored to intact status after insertion of the implant. There were no significant changes in left or right bending after discectomy or after implant insertion. Overall, the in vitro biomechanical function of the functional spine units with the Nubac was similar to the intact functional spine unit.

\section{Expulsion Tests}

Implant expulsion did not occur for any of the 6 samples in the first study. In the second study, no implant expulsion occurred during hand-bending with an annular window of $8 \mathrm{~mm} \ldots 10$ $\mathrm{mm}$. After the annulus was dissected, no implant expulsed under lateral bend testing on the machine for at least 8000 cycles.

\section{Wear Tests}

Results from the first test showed an average total mass loss of $2.79 \mathrm{mg} \pm 0.14 \mathrm{mg}$ at 10.35 million cycles. The corresponding average wear rate was $0.28 \mathrm{mg} \pm 0.07 \mathrm{mg} / \mathrm{million}$ cycles. The wear rate remained the same $(0.27 \mathrm{mg} \pm 0.09 \mathrm{mg} / \mathrm{million}$ cycles) after the device rotated $90^{\circ}$ into the lateral motion. Total mass loss was $5.5 \mathrm{mg}$ at 20.5 million cycles for both the flexion/extension and lateral bending tests. Even up to 40 million cycles, the wear rate remained the same. No failures of the test implants occurred throughout the test period and all implants functioned according to their design under the tested conditions in this study.

The results of the laser diffraction analysis for flexion/extension showed a volumetric diameter mean of $17.5 \mu \mathrm{m}$ and a number diameter mean of $0.86 \mu \mathrm{m}$. For lateral bending, the results showed a volumetric diameter mean of $37.3 \mu \mathrm{m}$ and number diameter mean of $0.48 \mu \mathrm{m}$. The results of the scanning electron microscopy analysis showed the particles had an average diameter of $0.78 \mu \mathrm{m}$ and were round to slightly elongated with an aspect ratio of 1.34 for flexion/extension testing.
The wear rate for the coupled motion test was $0.5 \mathrm{mg} / \mathrm{million}$ cycles, which compares very favorably to the wear rate of 10 $\mathrm{mg} / \mathrm{million}$ cycles for ProDisc (Synthes, West Chester, Pa) with the same ISO standard. ${ }^{11}$

\section{Static and Fatigue Strength Test}

The results of the static and fatigue strength tests showed a mean axial static load at offset yield of 10,427 N, which is well beyond the static failure strength of the human vertebral body or endplate. The failure mode was excessive plastic deformation of the top shell in all implants. The results of the dynamic axial fatigue test showed that the device had excellent axial fatigue strength. Run-out to 10 million cycles was achieved at $80 \%$ $(8342 \mathrm{~N})$ and $90 \%(9384 \mathrm{~N})$ of the average static offset yield load of $10,427 \mathrm{~N}$. At offset yield, 1 implant ran out to 10 million cycles whereas 1 implant failed immediately.

\section{Animal Study}

A limitation of this study was the use of a single Nubac height of $6 \mathrm{~mm}$ for all treated discs, which led to a significant increase of disc heights. The mean preoperative height of $5.61 \mathrm{~mm} \pm$ $0.88 \mathrm{~mm}$ was significantly increased to $6.41 \mathrm{~mm} \pm 0.83 \mathrm{~mm}$ at the postoperative interval $(P<.05)$. There was no significant change in disc height between the preoperative and postoperative conditions for the control level. For the 6- and 12-month treatment groups, the operative and control-level intervertebral disc space heights were compared between preoperation and at necropsy. For the 6-month group, the preoperative disc height at the L3-L4 and L5-L6 levels averaged $4.5 \mathrm{~mm} \pm 0.62 \mathrm{~mm}$ and $5.6 \mathrm{~mm} \pm 0.73 \mathrm{~mm}$, respectively. At 6 months postoperative, the L3-L4 level decreased to $2.9 \mathrm{~mm} \pm 0.15 \mathrm{~mm}$ and the L5L6 level decreased to $3.7 \mathrm{~mm} \pm 0.66 \mathrm{~mm}$, both of which were statistically significantly less than the preoperative condition $(P<.05)$. At 12 months postoperative, the L3-L4 and L5-L6 levels decreased to $2.7 \mathrm{~mm} \pm 0.28 \mathrm{~mm}$ and $3.5 \mathrm{~mm} \pm 0.92 \mathrm{~mm}$, respectively, which is statistically significantly less than in the preoperative conditions $(P<.05)$. The over-distraction resulted in difficulties for insertion and placement of the device. This may have resulted in the use of more force for proper placement and potentially induced endplate damage, Modic changes, and a corresponding decrease in disc height at the treated disc levels.

Multidirectional flexibility testing indicated diminished segmental motion for the Nubac under all loading modes. However, these findings were also noted in the operative control levels, demonstrating the effect of surgical intervention itself on segmental stability. By 12 months postoperative, flexion/ extension motion returned to the intact levels (Figure 5).

All treatments containing the Nubac implant exhibited increased densification of trabeculae along the endplate periphery, which corroborated with Modic Type 1 changes observed radiographically. These patterns of trabecular densification are 
Figure 5

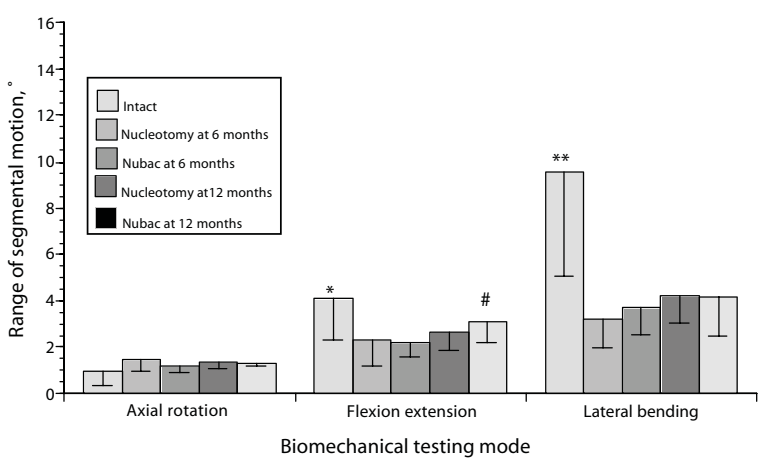

${ }^{*} \mathrm{P}<.05$ vs all other groups except those marked with a pound sign (\#); ${ }^{* *} \mathrm{P}<.05$ vs all other groups.

Range of motion of implant and control levels in the baboon study.

considered normal and have been documented in a number of in vivo nonhuman primate studies that investigated total disc arthroplasty devices. Evidence of mild implant subsidence was noted in 5 of 7 cases, which correlated with the absence of articular cartilage in subsidence regions. This may have been caused by over-distraction of the disc space leading to higher endplate stress. No change in densification or endplate sclerosis was observed at the L3-L4 surgical control levels. In the histological analyses, there was no detectable wear debris from the Nubac device and no evidence of macrophage activities or osteolytic response.

Pathological assessment for all undecalcified tissues assessed the following: tissue architecture, presence of debris, and signs of foreign body giant cell/granulomatous inflammatory reactions, degenerative changes, or autolysis. The histology demonstrated no significant evidence of any inflammatory reaction along the vertebral endplate. Pathological assessment characterized all systemic organs and organ systems as having normal tissue architecture, without presence of foreign body material, foreign body giant cell/granulomatous inflammatory reactions, degenerative changes, or autolysis. Plain and polarized light microscopy of local tissues overlying the operative sites from both the experimental and control levels indicated a chronic inflammatory reaction, with evidence of fibrous connective tissue replacement and infiltration of mononuclear cells. These observations were considered secondary to the surgical procedure and occurred at both the control and experimental operative levels.

A cytokine analysis showed no evidence of cellular apoptosis, giant cell reaction, or other significant pathological changes. Analysis of the immunohistochemical antibody stains for the local tissues overlying the experimental and control levels were negative in each case. There was no evidence of a proinflammatory cytokine reaction at any of the experimental or control levels.

\section{Clinical Study}

To assess the clinical utility of the Nubac at this early stage, the results - although still preliminary-included here describe clinical experience and data from an all-inclusive cohort from both outside and inside the United States as a part of the investigational device exemption feasibility study. At the time of publication, since December 2004, nearly 100 Nubac devices had been implanted. No major intraoperative or postoperative vascular or neurological complications occurred in this series. The preliminary clinical data showed significant decreases in both VAS and ODI scores after the Nubac procedure. The average preoperative VAS score was 76 , which decreased to 31 , 31, 31, 27, and 11 at 6 weeks, 3 months, 6 months, 12 months, and 24 months postoperative, respectively (Figure 6). Average ODI score decreased from 51 preoperatively to 31 ,

\section{Figure 6}

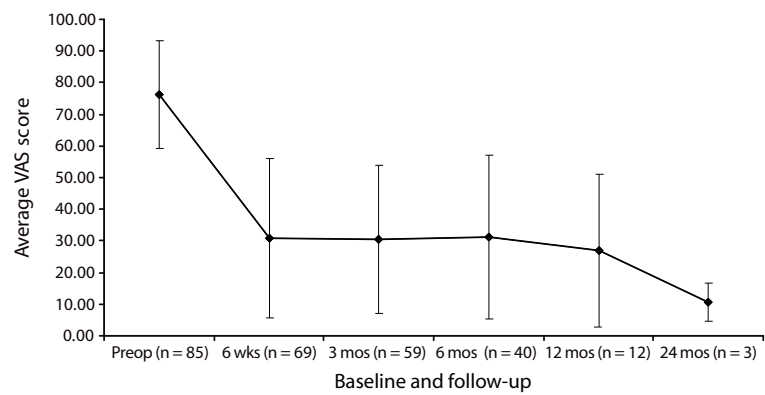

Visual Analog Scale (VAS) score improvement after Nubac implantation.

Figure 7

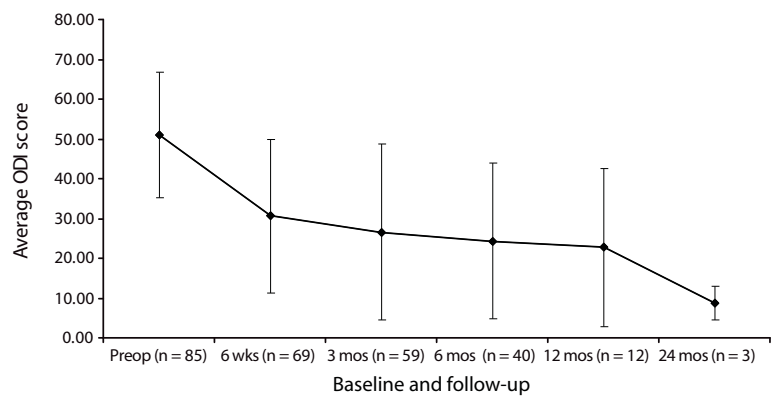

Oswestry Disability Index (ODI) score improvement after Nubac implantation. 
Figure 8

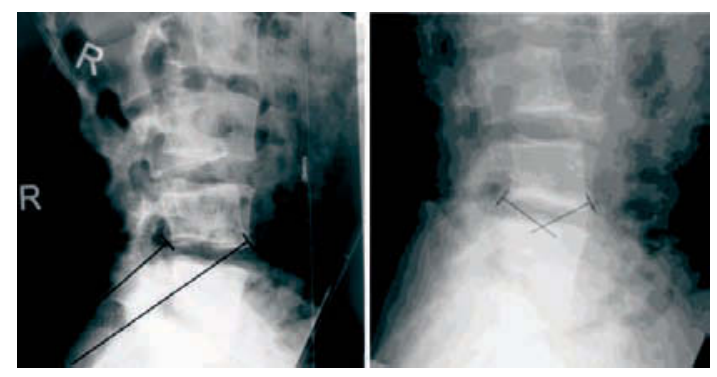

Center of rotation restoration after Nubac implantation: (a) before, (b) after.

27, 24, 23, and 9 at 6 weeks, 3 months, 6 months, 12 months, and 24 months postoperative, respectively (Figure 7). Because the Nubac implantation procedure preserves most of the disc tissues, such as annulus and ligaments, and restores the stability of the index segment, the preliminary in-vivo measurements of the center of rotation (COR) showed that the COR was restored to the normal location for a healthy disc (Figure 8).

\section{DISCUSSION}

Since its introduction to the medical market in 1999, PEEKOptima has quickly gained the confidence and acceptance of the medical community as a highly reliable and biocompatible material for permanent medical implants. Biodurability requirements for a permanent implantable device are largely dependent on its application. For implants such as total joints that are primarily used for older patients, a biodurability of 15 years would most likely be adequate, although with an increasing life expectancy and a progressively younger age group receiving these implants, biodurability requirements for these implants are likely to increase accordingly. However, for disc arthroplasty devices, which are used for much younger patients, the biodurability requirement should be much higher than that for total joints. Both in vitro and in vivo biodurability tests demonstrated that PEEK-Optima has an excellent biodurability for the disc arthroplasty application.

From the 2 biomechanical (kinematic) studies, it is clear that the Nubac was able to restore the normal ROMs and neutral zones in all 3 major rotational motions and restore the normal disc height in a human cadaveric spine model.

Because an intradiscal arthroplasty device is typically not fixed to the endplate, the potential for implant extrusion or expulsion has become a significant concern for this type of device. The only modern nucleus replacement with a fairly large number of clinical cases is the PDN-Solo prosthetic disc nucleus
(RayMedica, Minneapolis, Minn). Its initial poor clinical experience involved an implant expulsion rate that ranged from $8 \%$ to $36 \% .{ }^{12}$ This experience has heightened the concern about implant expulsion for all nucleus replacement devices.

In recognition of this potential risk, at the very beginning of the development of the Nubac, several human cadaver studies were conducted. We note that, because of the limited clinical experience with nucleus devices, no clinically validated standards have been established for assessing nucleus implant expulsion risk. Although the test has not clinically validated, it is believed that an in vitro cyclic bending test under physiological ROM is clinically relevant in assessing this risk. Unilateral bending opposite the annular window (through which the Nubac was inserted) was selected as the worst-case expulsion scenario.

The 2 cadaver expulsion tests demonstrated that the implant expulsion risk associated with Nubac is low. This low risk could be attributed to the inner articulation design, which allows the 2 plates to rotate along with the segment during rotation, keeping both plates in full contact with the endplates. The inner articulation design also helped the implant remain in the center of the disc even without annular constraint.

Because the Nubac has a ball-and-socket articulating joint and is made of a novel PEEK-on-PEEK self-mating material couple for load-bearing arthroplasty application, extensive wear testing has been conducted. To understand the wear characteristics of PEEK on PEEK as a self-mating material couple and to be able to compare it with the wear characteristics of other bearing materials, several wear tests with different methodologies were conducted. Because of the relatively short history of intradiscal arthroplasty, no established testing standards or guidelines exist to give an accurate and clinically validated biomechanical and wear assessment of the functionality of nucleus replacement devices. For this reason, and given the lack of clinical retrievals, only an educated assumption can, at best, be made. Because an intradiscal arthroplasty device is designed to share compressive loads with the remaining annulus, a dynamic compressive load that represented the range of loading according to the literature (based on the nucleus supporting about one half of the compressive load) was utilized in all testing methodologies. ${ }^{1}$

Overall, the wear rate for each individual sample over the entire testing period was consistent, suggesting that little variation arose among the samples. The first wear test also demonstrated that the wear rate is relatively insensitive to bidirectional motion. To our knowledge, the Nubac is the only disc arthroplasty device with a ball-and socket-articulation that has been tested to 40 million cycles while maintaining full functionality. 
Static and fatigue failure loads with the Nubac were much higher than the static and fatigue strengths of the vertebrae. Therefore, the results of these tests suggested that the Nubac has sufficient static and fatigue strengths for the intended application.

The biocompatibility results of the animal study serve as a scientific basis for ongoing clinical investigations into the use and efficacy of the Nubac for replacement of the intervertebral nucleus pulposus.

The main indication for the Nubac intradiscal arthroplasty device is discogenic back pain caused by DDD with or without leg pain, similar to that for interbody fusion and total disc arthroplasty devices. The main indication difference between intradiscal arthroplasty, or nucleus replacement, and interbody fusion/total disc arthroplasty is the requirement for a certain minimum disc height. The main reason for this disc height requirement is the clinical objectives mentioned previously: to restore/maintain the disc height and natural load sharing between the nucleus and annulus to achieve mechanical stability. If the disc is already too collapsed, an intradiscal arthroplasty device has to overstretch the annulus, which increases tension to regain the normal disc height and have the intradiscal arthroplasty device take all or the majority of the compressive load. In that case, the contact stress between the device and the endplates would be unphysiologically (abnormally) high and would lead to subsidence.

If one uses disc height as an indicator for the stage of disc degeneration cascade, significant disc height loss typically represents the late stage of disc degeneration. Therefore, intradiscal arthroplasty is more adequately indicated for patients at the early-to-moderate stage of the disc degeneration cascade, whereas fusion and total disc arthroplasty, because of their invasiveness and more "bridge-burning" nature, are appropriate for patients at the late stage of the cascade. But stage of degeneration should not be confused with the degree of pain - the early-to-moderate stage of the degeneration cascade does not involve less pain than in the late stage of the cascade. Patients with early-to-moderate disc degeneration can have as much pain, if not more, than patients at the late stage of degeneration.

In addition to being less invasive and less bridge-burning than total disc arthroplasty, intradiscal arthroplasty has the advantage of compatibility with different surgical approaches. In the limited clinical experience so far, the Nubac has been successfully implanted via all 3 common surgical approaches: posterior, straight lateral, and retroperitoneal anterolateral. Other than the differences in patient position, tissue dissection, and retraction for these 3 different approaches, the general surgical procedure for the Nubac for the 3 approaches is very similar.
The posterior approach is often used for patients with sequestrated or large contained disc herniation. Patient position, skin incision, and the approach to the disc are almost identical to that of discectomy. Depending on the pathology, the surgeon can approach the disc from either the left side or right side. Like a discectomy procedure, a partial laminectomy may be required, so that there will be enough window to conduct the discectomy. Although it is acceptable to remove a small edge of the facet in the medial side, care should be taken not to dissect too much facet. A nerve retractor should be used during the discectomy and device implantation.

For discs at L4-L5 or above, the straight lateral approach can be used. After positioning the patient in a lateral position, the disc is approached through a lateral retroperitoneal, transpsoatic pathway. Although this has not been a conventional approach for discectomy and interbody fusion, it has gained some popularity recently both for implanting nucleus devices ${ }^{13}$ and for interbody fusion. ${ }^{14}$ Compared with the conventional retroperitoneal anterolateral approach, this approach has the advantages of allowing anterior access to the disc space without a general surgeon or avoiding the potential complications of an anterior intra-abdominal procedure. For an intradiscal arthroplasty device, this approach also has the advantage of easy cleaning of the nucleus space. Alternatively, the conventional retroperitoneal anterolateral approach can be used. The disc can be approached from either side. Although the surgical approach is similar to that for total disc implantation and anterior interbody fusion, because of the small size of the Nubac implant, the area of disc exposure is much less than that for fusion and total disc implantation. With less tissue retraction and less bleeding, the risk of vascular injury and scar tissue formation should also be less.

Although the clinical data available are still preliminary, initial results are very encouraging. The absence of any major intraoperative and postoperative vascular and neurological complications supports the design rationale of the Nubac as less invasive and of less surgical risk. The initial effectiveness data as seen in the significant VAS and ODI improvements also suggest that the Nubac could be a viable treatment option for patients with low back pain caused by DDD.

Qi-Bin Bao, PhD, Matthew Songer, MD, Luis Pimenta, MD, $\mathrm{PhD}$, Dieter Werner, MD, Alejandro Reyes-Sanchez, MD, Massimo Balsano, MD, Umberto Agrillo, MD, Domagoj Coric, MD, Kenneth Davenport, MD, and Hansen Yuan.

From Pioneer Surgical Technology, Marquette, Mich (Bao); Instituto de Ortopedia, Mexico City, Mexico (Reyes-Sanchez); Orthopedic Surgery Association of Marquette, Marquette (Davenport); Santa Rita Hospital, Sao Paulo, Brazil (Pimenta); Carolina Neurosurgery and Spine Association, Charlotte, NC (Coric); Ospedale Sandro Pertini, Rome, Italy (Agrillo); UO 
Ortopedia Traumatologia, Thiene, Italy (Balsano); Arkade Privatklinik Niederschmalkalden, Breitungen, Germany (Werner); SUNY Upstate Medical University, Syracuse (Yuan).

Chip Bao is employed by Pioneer Surgical Technology, Marquette, Mich. Matthew Songer, Kenneth Davenport, and Hansen Yuan sit on the board of directors of Pioneer Surgical Technology.

Address correspondence and reprint requests to Qi-Bin (Chip) Bao, PhD, Pioneer Surgical Technology, 375 River Park Circle, Marquette, MI 49855 (email: chipbao@pioneersurgical.com).

This submission was received January 4, 2007, and accepted for publication February 26, 2007.

All collaborative studies were funded by Pioneer Surgical Technology, Marquette, Mich.

The authors would like to acknowledge the contributions from the following individuals and research institutes for their collaborations in several studies mentioned in this manuscript: Bryan Cunningham, MS (Union Memorial Hospital, Baltimore, Md), for the first kinematic study and the animal study; Nathanial Ordway, MS (State University of New York, Syracuse, NY), for the second kinematic study and the first expulsion study; Vijay Goel, PhD (University of Toledo, Toledo, Ohio), for the second expulsion study; and Thorsten Schwenke (Rush University Medical Center, Chicago, Ill) for the coupled motion wear test.

Institutional approval was obtained before the US IDE study.

\section{REFERENCES}

1. Nachemson A. The load on lumbar disks in different positions of the body. Clin Orthop Relat Res. 1966;45:107-122.

2. Blumenthal S, McAfee PC, Guyer RD, et al. A prospective, randomized, multicenter Food and Drug Administration investigational device exemptions study of lumbar total disc replacement with the Charité artificial disc versus lumbar fusion Part I: Evaluation of clinical outcomes. Spine. 2005;30:15651575.

3. McAfee PC, Cunningham B, Holsapple G, et al. A prospective, randomized, multicenter Food and Drug Administration investigational device exemption study of lumbar total disc replacement with the Charité artificial disc versus lumbar fusion Part II: Evaluation of radiographic outcomes and correlation of surgical technique accuracy with clinical outcomes. Spine. 2005;30:1576-1583.

4. Errico TJ. Lumbar disc arthroplasty. Clin Orthop Relat Res. 2005;(435):106-117.
5. Fernstrom U. Arthroplasty with intercorporal endoprosthesis in herniated disc and in painful disc. Acta Chir Scand Suppl. 1966;357:154-159.

6. McKenzie AH. Fernstrom intervertebral disc arthroplasty: a longterm evaluation. Ortho Int Ed. 1995;3:313-324.

7. International Organization for Standardization. ISO 10993-1 Biological evaluation of medical devices-Part 1: Evaluation and testing. 2003. Available at: http://www.iso.org.

8. Cartwright $\mathrm{K}$, Devine J. Investigation into the effect of $\mathrm{g}$ sterilization $(200 \mathrm{kGy})$ and accelerated aging on the properties of PEEK-Optima [technical report]. West Conshohocken, Pa: Invibio; 2005.

9. International Organization for Standardization. ISO/DIS 181921 Implants for surgery - Wear of total intervertebral spinal disc prostheses-Part 1: Loading and displacement parameters for wear testing and corresponding environmental conditions for tests. 2004. Available at: http://www.iso.org.

10. Fairbank JC, Pynsent PB. The Oswestry Disability Index. Spine. 2000;25:2940-2952.

11. Wright TM, Cottrell JM, Punga K, Rawlinson JJ, Gunsallus KL, Bartel DL. Retrieval and wear analyses of ProDisc lumbar disc implants. Presented at: 6th Annual Meeting of the Spine Arthroplasty Society, May 9-13, 2006, Montreal, Québec.

12. Klara PM, Ray CD. Artificial nucleus replacement: clinical experience. Spine. 2002;27:1374-1377.

13. Bertagnoli R, Vazquez RJ. The Anterolateral TransPsoatic Approach (ALPA): a new technique for implanting prosthetic disc-nucleus devices. J Spinal Disord Tech. 2003;16:398-404.

14. Ozgur BM, Aryan HE, Pimenta L, Taylor WR. Extreme Lateral Interbody Fusion (XLIF): a novel surgical technique for anterior lumbar interbody fusion. Spine J. 2006;6:435-443. 